\title{
DEFECTS IN SOME HYDROGENATED METALLIC MATERIALS STUDIED BY POSITRONS
}

\author{
V.S. Mikhalenkov \\ Institute of Metal Physics, National Academy of Sciences of Ukraine \\ Vernadskii str., 36, 03680 Kiev-142, Ukraine
}

\begin{abstract}
The measurements of angular correlation of annihilation radiation and positron lifetime were performed to study defects in commercial stainless steel and nickel subjected to deformation and electron irradiation. It follows from variation of $S$-parameter derived from angular correlation of annihilation radiation spectra that hydrogen charging of $\mathrm{Fe}-\mathrm{Cr}-\mathrm{Ni}-\mathrm{Mn}$ austenite steel results in appearance of modest amount of vacancies which agglomerate into small clusters during annealing at low temperatures. Electron irradiation produces much more defects, so that subsequent hydrogenation does not add much to their concentration. The major part of defects anneals out gradually to $700 \mathrm{~K}$. The residual part is preserved up to $950 \mathrm{~K}$. This fact testifies to bonding state of vacancies. Nickel of $3 \mathrm{~N}$ purity was prepared in three initial states, i.e. cold-rolled and hydrogenated before and after deformation. In an initial state all samples contain, along with simple defects, vacancy clusters. Their size and concentration are much larger in hydrogenated samples, as compared with merely cold-rolled one. Annealing above $400 \mathrm{~K}$ causes growth of cluster size and decrease in their concentration. At $500 \mathrm{~K}$ clusters are observed to disappear in cold-rolled sample, while in the sample hydrogenated after deformation they survive to $600 \mathrm{~K}$.
\end{abstract}

PACS numbers: $78.70 \mathrm{Bj}$

\section{Introduction}

Hydrogen strongly affects the properties of metallic materials. On frequent occasions it exerts harmful influence on mechanical properties. For hydride forming metals hydrogen embrittlement arises from precipitation of hydrides at the grain boundaries. In the case that stable hydrides do not exist, the changes of properties may be caused by interaction of hydrogen with another defects of crystalline structure. In particular, interaction of hydrogen atoms with vacancies can promote nucleation of cavities which are capable of forming cracks. That is why hydrogen interaction with vacancies is of marked interest.

Positron spectroscopy has proved to be a unique tool in studying vacancy type defects in solids. It was repeatedly utilized in research of hydrogen behaviour 
in metals. One of the first paper on this point was that by Lengeler et al. [1] who have shown that hydrogen in $\mathrm{Cu}$ was trapped by vacancies and did not escape up to $450 \mathrm{~K}$. Hydrogenated $\mathrm{Ni}$ and its alloys were an object of a number of papers [1-6]. Particularly, Pająk and Rozenfeld [4] noticed enhancing effect of hydrogenation on the deformation induced changes in peak intensity of angular correlation of annihilation radiation (ACAR) and the difference in annealing behaviour of deformed $\mathrm{Ni}$ with and without hydrogen. The idea was proposed that hydrogen initiated vacancy clustering. The effect of hydrogen on the defect structure in Ni-based alloys was studied in [7]. However, despite all efforts many questions on how hydrogen affects the formation of defects in metal still leave unclearified.

In this paper the measurements of ACAR and positron lifetime (PLT) have been performed to study defects in commercial stainless steel and nickel subjected to hydrogen charging combined with deformation and electron irradiation.

\section{Investigation of stainless steel}

\subsection{Experimental procedure}

Austenite $\mathrm{Fe}_{56} \mathrm{Cr}_{18} \mathrm{Ni}_{16} \mathrm{Mn}_{10}$ stainless steel was produced by argon-arc melting. Hydrogenation of such steel is known to promote formation of $\varepsilon$-phase. $\mathrm{Ni}$ trogen is known to suppress this effect and stabilize fcc $\gamma$-phase [8]. Therefore the prepared steel was doped with $0.07 \% \mathrm{~N}$ by plasma-arc remelting. Sample preparation included cutting and quenching in water after keeping for $15 \mathrm{~min}$ at $1273 \mathrm{~K}$ in purified Ar. Initial state was reached by annealing in Ar at $1223 \mathrm{~K}$ during $3 \mathrm{~h}$. After this the samples were subjected to different treatments, namely:

(i) hydrogen charging by electrolytic method in $1 \mathrm{~N}_{2} \mathrm{SO}_{4}$ water solution with addition of $250 \mathrm{mg} \mathrm{l}^{-1}$ of $\mathrm{NaAsO}_{2}$ as recombination poison, at current density $500 \mathrm{~mA} \mathrm{~cm}^{-2}$ during $64 \mathrm{~h}$ (sample $\mathrm{H}$ );

(ii) electron irradiation $\left(3 \mathrm{MeV}, 10^{18} \mathrm{e}^{-} \mathrm{cm}^{-2}\right.$ ) with subsequent hydrogenation (sample EH);

(iii) cold rolling to $34 \%$ thickness reduction (sample D).

ACAR spectra were taken using conventional long-slit device with angular resolution of $0.7 \mathrm{mrad}$. The shape of ACAR was analyzed in terms of $S$-parameter defined as the normalized area in a fixed number of channels in central part of spectrum being chosen so as to get for well-annealed sample $S$ value close to 0.5 . $S$-parameter was measured as a function of isochronal $(2 \mathrm{~h})$ annealing temperature. The anneal was performed in vacuum of $0.26 \mathrm{mPa}$. Before the first measurement hydrogenated samples were exposed at air during $72 \mathrm{~h}$.

\subsection{Results}

The dependence of $S$-parameter on annealing temperature is shown in Fig. 1. It is seen that any of performed treatments results in increase in $S$-parameter, with respect to that for well-annealed state, but the magnitudes of increase are distinct for different treatments. Only $3 \%$ growth of $S$-parameter is produced by hydrogenation, whereas the effect of cold-rolling amounts to $15.4 \%$. Intermediate value of $S$-parameter rise $(9.8 \%)$ is found after electron irradiation. It is worth 


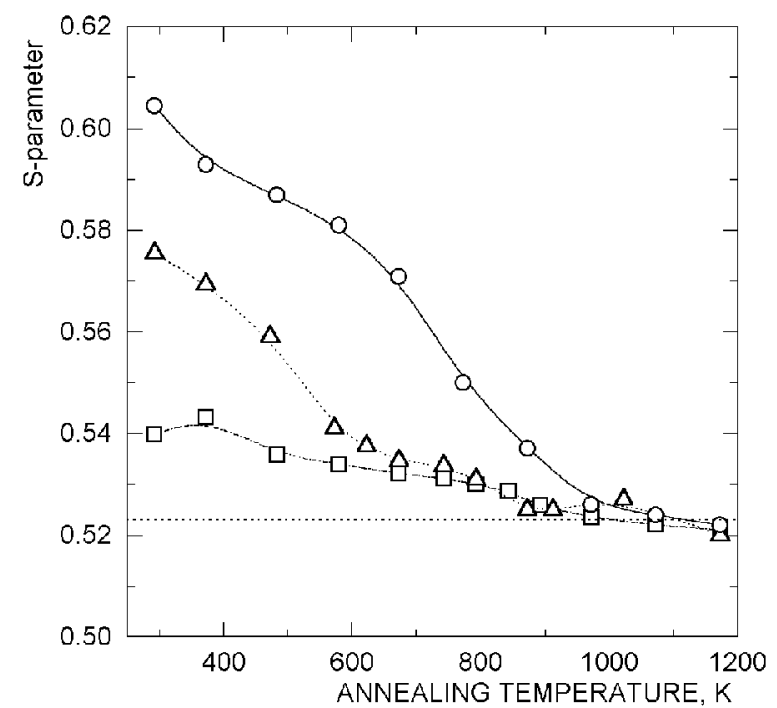

Fig. 1. Variation of $S$-parameter in deformed (circles), hydrogenated (squares) and charged with hydrogen after electron irradiation (triangles) stainless steel with annealing temperature. Horizontal dotted line indicates $S$ value for annealed steel. The lines are guides to an eye.

mentioning that hydrogenation of irradiated sample does not change $S$-parameter. When being annealed, the samples gradually return to annealed state and reach it at about $950 \mathrm{~K}$. Only in H-sample small hump is seen at $370 \mathrm{~K}$. The recovery stages are rather blurred. In D-sample one can distinguish two stages with different recovery rates. In EH-sample the major portion of $S$-parameter growth vanishes near $600 \mathrm{~K}$.

\subsection{Discussion}

Vacancies in pure iron are known to become movable at $220 \mathrm{~K}$ [9]. Their conservation up to considerably higher temperatures gives evidence that they are in the bonded state. But blurring of $S$-parameter reduction with annealing temperature suggests that vacancy bonds are of great variety and are broken at different temperatures. This is not particularly surprising taking into account complicated composition of the steel involved.

Small effect of hydrogenation indicates that defect concentration initiated by hydrogen is minor. Moderate hump of $S$-parameter at $300 \mathrm{~K}$ can be conceded to come from formation or growth of small vacancy clusters.

As electron irradiation produces vacancy-interstitial atom pairs, the recovery stage which proceeds up to $570 \mathrm{~K}$, should be conditioned by moving out of vacancies. Similar stage is revealed in D-sample. More sharp decrease in $S$-parameter observed in this sample above $670 \mathrm{~K}$ can be attributed to recrystallization processes. The nature of small reduction in S-parameter seen in $\mathrm{H}$ - and EH-samples near $900 \mathrm{~K}$ is not clear. 
The main feature of results obtained for stainless steel is insignificant effect of its charging with hydrogen. Even if vacancy clusters are really formed, their size is too small to initiate the cracks. This makes us conclude that hydrogen embrittlement of this type of steel might be caused by formation of hydrogen containing phases. In our case the formation of such $\varepsilon$-phase is suppressed by doping with nitrogen, and hydrogen remains in austenite lattice. This conclusion is consistent with the idea of the improving action of nitrogen on mechanical properties of $\mathrm{Fe}-\mathrm{Cr}-\mathrm{Ni}-\mathrm{Mn}$ steel [8]. On the other hand, in some fcc metals, particularly in $\mathrm{Ni}$, the effect of hydrogen on annihilation parameters is much larger. This made us turn back to this well studied metal.

\section{Investigation of nickel}

\subsection{Experimental procedure}

The samples were made from $3 \mathrm{~N}$ nickel containing $\mathrm{Fe}(0.02 \%), \mathrm{Cu}(0.01 \%)$, Co $(0.02 \%), \mathrm{O}(0.026 \%)$ and $\mathrm{N}(0.017 \%)$ as the main impurities. After cold-rolling to the needed thickness the samples were annealed at $1270 \mathrm{~K}$ during $2 \mathrm{~h}$ in Ar atmosphere in order to obtain the reference state. Thereafter three kinds of samples were prepared:

(i) deformed by cold-rolling to the thickness reduction of $30 \%$ (sample D);

(ii) deformed in the same way and then charged with hydrogen by means of the method described in Sec. 2.1 (sample DH);

(iii) hydrogenated in annealed state and then deformed (sample HD).

The samples were subjected to isochronal annealing in vacuum of $0.26 \mathrm{mPa}$ at sequentially increasing temperatures up to $825 \mathrm{~K}$. Each treatment was followed by measurements of positron lifetime (PLT) and Doppler broadening of annihilation line (DBAL) spectra. Positron source of $2 \mathrm{MBq}{ }^{22} \mathrm{NaCl}$ sealed into kapton foil $1 \mathrm{mg} / \mathrm{cm}$ thick was sandwiched between two identical samples. PLT spectra were taken at room temperature using conventional fast-fast coincidence system with a time resolution of 240 ps (FWHM). More than $2 \times 10^{6}$ events were accumulated in each spectrum. After background and source corrections, the spectra were analyzed by taking use of POSITRONFIT programme [10]. The attempt to use three-component analysis has failed. Only two components were revealed in all spectra. In the annealed sample, along with lifetime of $110 \mathrm{ps}$, long-lived component of about $350 \mathrm{ps}$ with the intensity of $1.5 \%$ is revealed. In future analysis this component is disregarded. All other PLT spectra up to $900 \mathrm{~K}$ contain intermediate components close to $166 \mathrm{ps}$ that coincide with PLT for vacancy in $\mathrm{Ni}$ [6]. This fact gave ground to fix PLT value of $166 \mathrm{ps}$. The average lifetime was calculated as $\tau_{\text {av }}=I_{1} \tau_{1}+I_{2} \tau_{2}$, where $\tau_{1}$ and $\tau_{2}$ are PLT components and $I_{1}$ and $I_{2}$ are their intensities. DBAL spectra were measured using intrinsic Ge detector with energy resolution of $1.24 \mathrm{keV}$ at ${ }^{103} \mathrm{Ru}$ peak. The position of annihilation peak was stabilized by monitoring the system with ${ }^{103} \mathrm{Ru} \gamma$-line. DBAL line shape was analyzed in terms of $S$-parameter determined as the normalized area in a fixed number of channels in the central part of spectrum. The number of fixed channels was chosen so as to get for as-quenched samples $S$ value close to 0.5 . 


\subsection{Results}

Figure 2 displays the dependences of $\tau_{\mathrm{av}}$ and $S$ on the annealing temperature. The effect of hydrogen charging is seen to be very strong. For all samples two main stages of recovery can be distinguished. Their temperature ranges correlate with those observed in deformed $\mathrm{Ni}$ [11]. At the first stage the behaviour of $\tau_{\mathrm{av}}$ and $S$ in DH and HD samples is practically similar irrespective to the sequence of
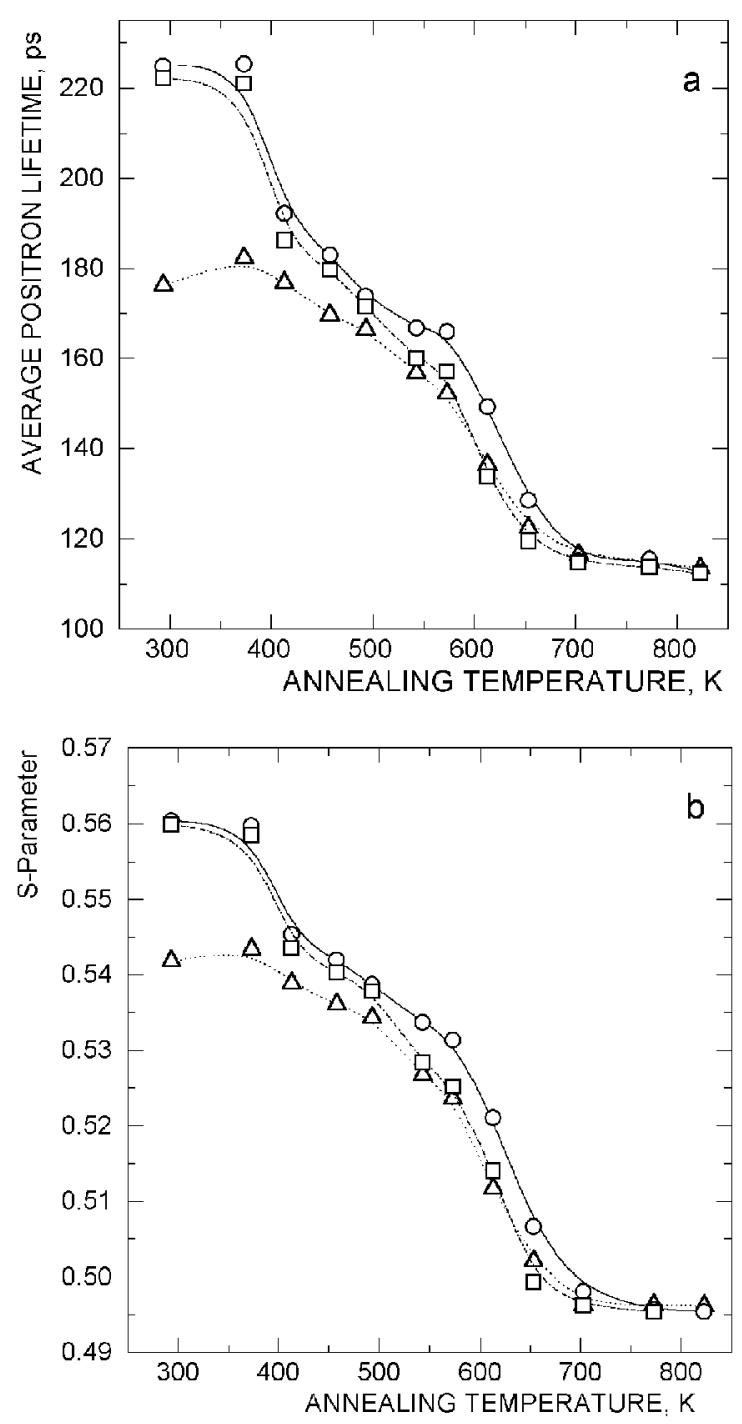

Fig. 2. Average positron lifetime (a) and $S$-parameter (b) in deformed (triangles) and hydrogenated before (squares) and after (circles) deformation nickel vs. annealing temperature. The lines are guides to an eye. 

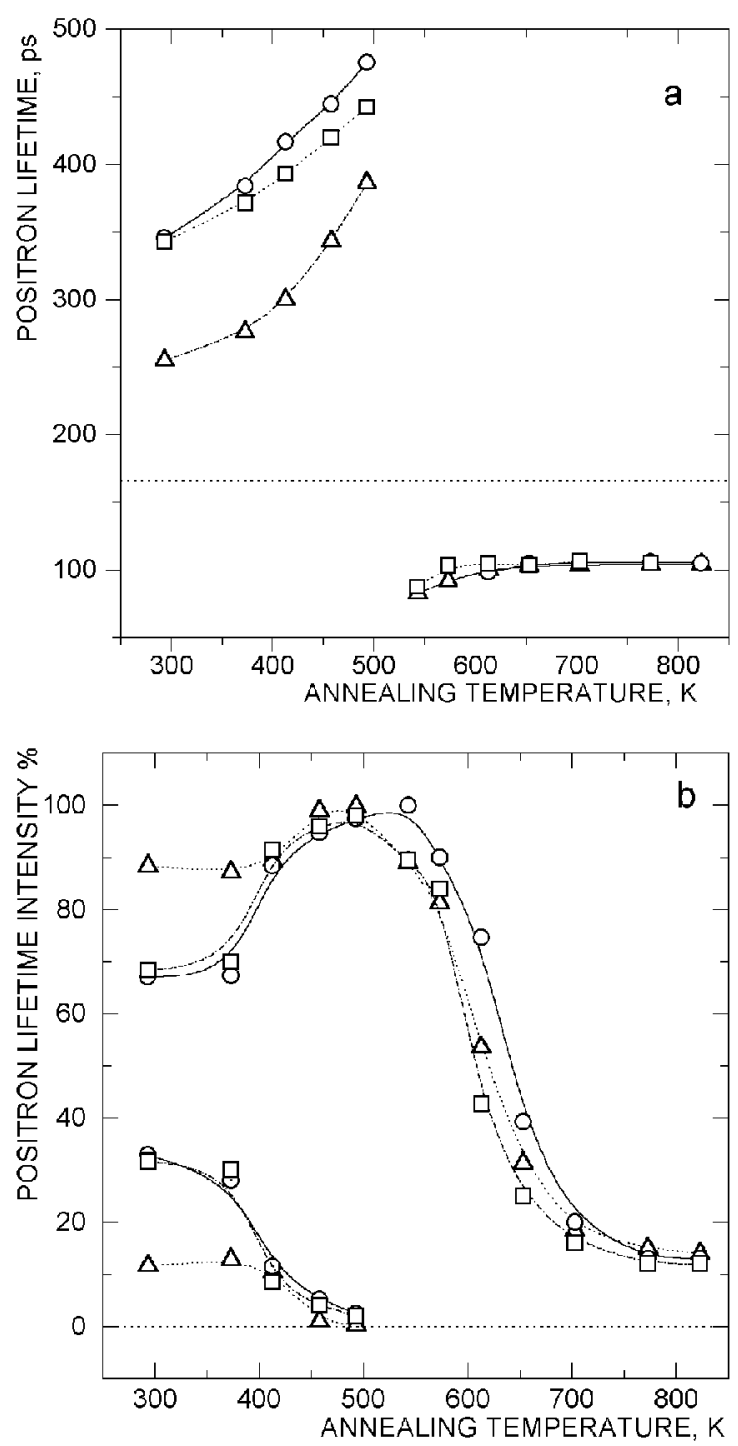

Fig. 3. Positron lifetime components (a) and intensities of long and fixed components (b) in deformed (triangles) and hydrogenated before (squares) and after (circles) deformation nickel vs. annealing temperature. Horizontal dotted line shows the value of fixed lifetime. The lines are guides to an eye.

hydrogenation and deformation. At the second stage (above $550 \mathrm{~K}$ ), which is considered to be caused by recrystallization, recovery process in D- and HD-samples proceeds more rapidly than in DH one. At $700 \mathrm{~K} \tau_{\text {av }}$ and $S$ return to the values peculiar to reference sample.

The results of PLT spectra analysis are plotted in Fig. 3. One of the features of this figure is PLT of $250 \mathrm{ps}$ with the intensity about $10 \%$ in D-sample 
in as-deformed state. At the same time in HD- and DH-samples this component amounts to $350 \mathrm{ps}$ and its intensity is of $30 \%$. Above $370 \mathrm{~K}$ long PLT in all samples begins to rise and its intensity starts to drop and vanishes in the range of 500-550 K. Simultaneously short PLT component of about 80 ps comes into view and is gradually increasing in both intensity and magnitude reaching $105 \mathrm{ps}$ the value typical of annihilation in Ni bulk.

\subsection{Discussion}

The most interesting features of the obtained results are the presence of vacancy clusters in as-deformed sample, much larger size and concentration of clusters in hydrogenated ones and their higher stability in the sample charged after deformation. As the purity of $\mathrm{Ni}$ used was not very high, some impurity atoms might initiate agglomeration of vacancies promoting cluster formation. However, hydrogen atoms do better in this respect. According to theoretical calculations [12], PLT of 250 ps corresponds to the clusters consisting of 4 vacancies, while PLT of 350 ps observed in hydrogenated samples is peculiar to agglomeration of more than 10 vacancies. Above $370 \mathrm{~K}$ vacancies in $\mathrm{Ni}$ get movable [11] and enable clusters to grow incorporating single vacancies or providing process of coalescence. Above $500 \mathrm{~K}$ clusters start to dissociate, and vacancies start to migrate to the sinks. It is not clear why this process is delayed in DH-sample with respect to HD one. In fact, defect structure in DH- and HD-samples are formed under somewhat different conditions. In the course of charging hydrogen in HD-sample is being uniformly distributed over the perfect lattice, while in $\mathrm{DH}$ one its distribution depends on defects created by deformation. This may be one of the reasons for somewhat higher stability of defect structure in Ni deformed after hydrogenation, especially against recrystallization, though the mechanisms of the processes occurring are not known. Moreover, it is not clear why annihilation parameters in these two samples in pre-annealed state are nearly the same. Thus further research is needed to shed light on the role of hydrogen in evolution of defect structure of metals.

\section{Acknowledgment}

The author is very grateful to Drs. Y.N. Jagodzinski and A.V. Tarasenko for providing of samples processing and to Profs. A.P. de Lima and C. Lopes Gil for promoting positron lifetime measurements.

\section{References}

[1] B. Lengeler, S. Mantl, W. Triftshäuser, J. Phys. F 8, 1691 (1978).

[2] C.E. Price, L.B. Traylor, Scr. Met. 17, 901 (1983).

[3] C.S. Sundar, A.B. Bharathi, K.P. Gopinathan, Philos. Mag. A 50, 635 (1984).

[4] J. Pająk, B. Rozenfeld, in: Positron Annihilation, Eds. P.S. Jain, R.M. Singru, K.P. Gopinathan, World Sci., Singapore 1985, p. 558.

[5] S. Chabik, Phys. Status Solidi A 120, K125 (1990). 
[6] Cs. Czeles, G. Lang, Zs. Kajcsos, Mater. Sci. Forum 105/110, 1261 (1992).

[7] K.D. Moor, F.H. Cocks, P.L. Jones, J. Test Eval. 11, 309 (1983).

[8] V.G. Gavriljuk, H. Hänninen, A.V. Tarasenko, A.S. Tereshchenko, K. Ullakko, Acta Metall. Mater. 43, 559 (1995).

[9] P. Hautojärvi, T. Judin, A. Vehanen, J. Yli-Kauppila, J. Verdone, P. Moser, Solid State Commun. 29, 855 (1979).

[10] P. Kirkegaard, M. Eldrup, O.E. Mogensen, N.J. Pedersen, Comput. Phys. Commun. 23, 307 (1981)

[11] G. Dlubek, O. Brümmer, N. Meyendorf, J. Phys. F 9, 1961 (1979).

[12] M. Puska, R.M. Nieminen, J. Phys. F 13, 333 (1983). 